Original Article

\title{
Use of phytase and citric acid supplementation on growth performance and nutrient digestibility of Cirrhinus mrigala fingerlings fed on canola meal based diet
}

\author{
Uso de suplementação de fitase e ácido cítrico no desempenho do crescimento e \\ digestibilidade de nutrientes de dedos Cirrhinus mrigala alimentados em dieta à base \\ de refeição de canola
}

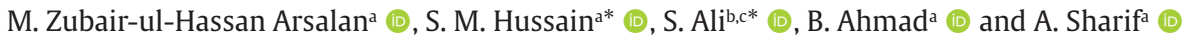 \\ aGovernment College University, Department of Zoology, Fish Nutrition Lab, Faisalabad, Pakistan \\ ${ }^{\mathrm{b}}$ Government College University, Department of Environmental Sciences \& Engineering, Faisalabad, Pakistan \\ ${ }^{c}$ China Medical University, Department of Biological Sciences and Technology, Taichung, Taiwan
}

\begin{abstract}
Fishmeal; being a limited and costly feed ingredient is continuously been substituted with locally available plant proteins. However, the occurrence of anti-nutritional factors in plant meal suppresses its potential to be fully replaced. Therefore, in this study we aimed to study the synergistic effects of dietary additives like citric acid and phytase enzyme supplementation on growth performance and nutrient digestibility of Cirrhinus mrigala fingerlings. Canola meal (CM) was used as a test ingredient to replace fishmeal (FM) as; $0 \%, 25 \%, 50 \%$ and $75 \%$. These four diets were further supplemented by varying levels of phytase $\left(0\right.$ and $\left.750 \mathrm{FTU} \mathrm{kg}^{-1}\right)$ and citric acid $\left(0 \%\right.$ and $2.5 \%$ ) to formulate total sixteen test diets as $\mathrm{T}_{1}, \mathrm{~T}_{2}, \mathrm{~T}_{3}, \mathrm{~T}_{4}, \mathrm{~T}_{5}, \mathrm{~T}_{6}, \mathrm{~T}_{7}, \mathrm{~T}_{8}, \mathrm{~T}_{9}, \mathrm{~T}_{10}, \mathrm{~T}_{11}, \mathrm{~T}_{12}, \mathrm{~T}_{13}, \mathrm{~T}_{14}, \mathrm{~T}_{15}$ and $\mathrm{T}_{16}$. Each treatment contained three replicates; applied to fish groups having 15 fingerlings each; following $3 \times 3$ factorial arrangement. $1 \%$ of chromic oxide was added as an inert marker. Maximum weight gain\% (288\%) and the lowest value of FCR (1.07) were recorded when fish was fed on diet $\mathrm{T}_{12}$ as compared to fish fed control diet $\left(\mathrm{T}_{1}\right)$. Similarly, optimum nutrient digestibility values such as crude protein (77\%), crude fat (84\%) and gross energy (70\%) were noted on same level. It was concluded that $50 \%$ canola meal can optimally replace fishmeal when supplemented with phytase and citric acid at the levels of $750 \mathrm{FTU} \mathrm{kg}^{-1}$ and $2.5 \%$, respectively.
\end{abstract}

Keywords: C. mrigala, growth, nutrient digestibility, fishmeal, canola meal.

\begin{abstract}
Resumo
A farinha de peixe, por ser um ingrediente alimentar limitado e caro, é continuamente substituída por proteínas vegetais disponíveis localmente. No entanto, a ocorrência de fatores antinutricionais na farinha de plantas suprime seu potencial de ser totalmente substituída. Portanto, neste estudo objetivamos estudar os efeitos sinérgicos de aditivos dietéticos como ácido cítrico e suplementação com enzima fitase sobre o desempenho de crescimento e digestibilidade de nutrientes de alevinos de Cirrhinus mrigala. A farinha de canola (CM) foi usada como ingrediente de teste para substituir a farinha de peixe (FM) como: 0\%, 25\%, 50\% e 75\%. Essas quatro dietas foram suplementadas por níveis variados de fitase ( 0 e $750 \mathrm{FTU} \mathrm{kg}^{-1}$ ) e ácido cítrico ( $0 \%$ e 2,5\%) para formular um total de 16 dietas de teste como T1, T2, T3, T4, T5, T6, T7, T8, T9, T10, T11, T12, T13, T14, T15 e T16. Cada tratamento continha três repetições; aplicado a grupos de peixes com 15 alevinos cada; seguindo o arranjo fatorial $3 \times 3.1 \%$ de óxido crômico foi adicionado como um marcador inerte. \% de ganho de peso máximo (288\%) e o valor mais baixo de $\operatorname{FCR}(1,07)$ foram registrados quando os peixes foram alimentados com dieta T12 em comparação com peixes alimentados com dieta controle (T1). Da mesma forma, valores ótimos de digestibilidade de nutrientes, como proteína bruta (77\%), gordura bruta (84\%) e energia bruta (70\%) foram anotados no mesmo nível. Concluiu-se que $50 \%$ da farinha de canola pode substituir de forma ideal a farinha de peixe quando suplementada com fitase e ácido cítrico nos níveis de 750 FTU $\mathrm{kg}^{-1}$ e $2,5 \%$, respectivamente.
\end{abstract}

Palavras-chave: C. mrigala, crescimento, digestibilidade de nutrientes, farinha de peixe, farelo de canola.

*e-mail: drmakhdoom90@gmail.com; shafaqataligill@gcuf.edu.pk

Received: December 14, 2020 - Accepted: January 20, 2021 


\section{Introduction}

Fishmeal is a vital ingredient being used in fish feed formulation (Salin et al., 2018). It is a main source of nutrients such as essential vitamins, amino acids, fatty acids and trace minerals. The intensive use of fishmeal has affected its protein quality as well as the cost. The need to replace fishmeal in aqua-feed is mandatory because the sustainability of boosting aquaculture industry requires the decrease in wild fish capture (Alhazzaa et al., 2019). Suitable plant feed ingredients such as grains and oilseed by-products meal are the most promising sources of protein and energy for feed formulation in the future (Manuel et al., 2019). Studies proved that fishmeal can be replaced up to $30 \%$ by an equal mixture of cottonseed and soybean meal; with iron and phytase in the presence of lysine and methionine in fish feed (Lim and Lee, 2009).

Canola meal (CM) is a nutritionally important feed ingredient derived from Brassica napus, containing 33-45\% protein contents (Glencross, 2016). It is less expensive than FM and ubiquitous in nature. It has met an elevated global production since last years. Being a highly suitable plant-based source of protein, it is incorporated as a major protein source in feed formulation (Mushtaq et al., 2006). The oilseed by-products meals are increasingly important in preparation of economical and environment friendly fish feed (Cheng and Hardy, 2002) but one major problem associated with the use of plant protein is the presence of anti-nutritional factors such as phytate, fibers and glucosinolates (von Danwitz and Schulz, 2020).

It is estimated that up to $80 \%$ of the total phosphorus contents in plants are present in the form of phytate and is practically not available for monogastric or agastric animals (NRC, 1993); due to lack of intestinal phytase (PHY) for efficient phytate hydrolysis during digestion (Jackson et al., 1996). It also makes a bond with vitamins and proteins and reduces their utilization, absorption and interferes with starch and lipid digestibility (Papatryphon et al., 1999). Studies have shown the efficacy of PHY as growth enhancer in species such as carps and Nile tilapia (Phromkunthong et al., 2010; Olusola and Nwanna, 2014).

It has been reported that citric acid (CA) is being successfully used to improve growth performance, nutrients utilization and resistance against diseases due to its phytate hydrolyzing capability (Dai et al., 2018). Being a chelating agent, it also works as a binder with various types of cations in the intestine and makes them easy to be absorbed by lowering the gut $\mathrm{pH}$ and improve bone mineralization (Shafique et al., 2018). A number of feeding trials with promising responses have been conducted to evaluate the effect of CA acidified diets on growth, feed intake and feed performance of fish (Pandey and Satoh, 2014; Bano and Afzal, 2018).

C. mrigala commonly known as "mori" is one of the major Indian carp species; cultured in Pakistan (Hussain et al., 2011). It is bottom feeder and feed on decaying organic matter and vegetable debris. For the success of carp farming, the use of cost-effective feed has become necessary to control the farm's economy (Abid and Ahmed, 2009). So, it is necessary to introduce nutritionally balanced diet as a limiting factor for carp's optimal growth (Khan et al.,
2004). Therefore, the present study was conducted to investigate the effect of CA and PHY supplementation on growth performance and nutrient digestibility of C. mrigala fingerlings fed CM based diets as well as to help the aqua-culturists in producing environment friendly and cost-effective fish.

\section{Materials and Methods}

The current experiment was conducted in Fish Nutrition Laboratory, Department of Zoology, Government College University Faisalabad, Pakistan (Latitude $31.4166^{\circ}$ North and the Longitude $73.0707^{\circ}$ East).

\subsection{Experimental design}

A 90 days, $3 \times 3$ factorial experiment was conducted by using canola meal as a test ingredient. Four test diets were formulated by replacing fishmeal with canola meal at levels of $0 \%, 25 \%, 50 \%$ and $75 \%$. These four diets were further supplemented by varying levels of phytase ( 0 and 750 FTU $\mathrm{kg}^{-1}$ ) and CA ( $0 \%$ and 2.5\%); to formulate total sixteen test diets, designated as $\mathrm{T}_{1}, \mathrm{~T}_{2}, \mathrm{~T}_{3}, \mathrm{~T}_{4}, \mathrm{~T}_{5}, \mathrm{~T}_{6}$, $\mathrm{T}_{7}, \mathrm{~T}_{8}, \mathrm{~T}_{9}, \mathrm{~T}_{10}, \mathrm{~T}_{11}, \mathrm{~T}_{12}, \mathrm{~T}_{13}, \mathrm{~T}_{14}, \mathrm{~T}_{15}$ and $\mathrm{T}_{16}$. Each treatment contained three replicates having 15 fingerlings (initial weight; $6.570 \pm 0.053 \mathrm{~g}$ ) in each triplicate tank, hence a total of 720 fingerlings were stocked.

\subsection{Feed formulation}

Feed ingredients including CM were procured from the local market and their chemical composition was analyzed before the formulation of the test diets by standard methods (AOAC, 2005). All the feed ingredients were grinded finely to pass through $(0.5 \mathrm{~mm})$ mesh size (Table 1$)$. An electric mixer was used to mix all feed ingredients for about 10-20 minutes and fish oil was added gradually while mixing. 15\% moisture was added by adding distilled water and pellets were made by using pelleting machine (Lovell, 1989). Preceding the pellet formation, phytase was supplemented by spraying method (Jackson et al., 1996). The pellets were then dried at room temperature and refrigerated at $4{ }^{\circ} \mathrm{C}$, until the start of feeding trial.

\subsection{Experimental facility and fish feeding}

C. mrigala fingerlings were obtained from Government Fish Seed Hatchery, Faisalabad. The fingerlings were acclimatized and stocked in V-shaped tanks with proper aeration provided in the laboratory conditions. During the period of acclamation, the fish were fed on basal diet once daily (Allan and Rowland, 1992). Before beginning of experiment, $C$. mrigala fingerlings were given the dip prophylactically in $\mathrm{NaCl}$ solution $(5 \mathrm{~g} / \mathrm{L})$ to remove the ectoparasites and to avoid from any fungal infection (Rowland and Ingram, 1991). During this experiment, triplicate tanks were allotted for each treatment. Fingerlings were fed by hand till apparent satiation at 07:00 a.m.; at a ration equal to $5 \%$ of its live wet weight. Uneaten feed was collected after an hour of feeding session and oven dried at $60^{\circ} \mathrm{C}$ for feed consumption analysis. After that, experimental tanks were washed and refilled with tap water. All tanks were aerated round the clock and water 
quality parameters were monitored and maintained by using physical equipment; including thermometer for temperature, DO meter (Jenway 970) for dissolved oxygen and $\mathrm{pH}$ meter (Jenway 3510) for $\mathrm{pH}$ measurements.

\subsection{Chemical analysis of feed and feces}

Feed ingredients, samples of prepared test diets and feces were homogenized with the help of motor and pestle, then subsequently analyzed by the given standard methods. Moisture was determined at $105^{\circ} \mathrm{C}$ for $12 \mathrm{~h}$ by oven drying method, crude protein $(\mathrm{N} \times 6.25)$ by micro-Kjeldahl apparatus, crude fat (Soxtec HT2 1045 system) by ether extraction method, crude fiber by loss on ignition and gross energy by oxygen bomb calorimeter.

\subsection{Growth study}

Variation in the growth parameters of $C$. mrigala fingerlings was estimated at the beginning and end of the experiment by following standard methods (Hussain et al., 2015) (Equations 1-3).

Weight gain $\%=\frac{(\text { Final weight }- \text { Initial weight })}{\text { Initial weight }} \times 100$

$$
\begin{aligned}
& F C R=\frac{\text { Total dry feed intake }(g)}{\text { Wet weight gain }(g)} \\
& S G R=\frac{\text { Final Weight }(g)-\text { Initial Weight }(g)}{\text { No of trial days }} \times 100
\end{aligned}
$$

\subsection{Digestibility study}

Preceding the oxidation with molybdate reagent, chromic oxide contents in diets and feces were estimated using UV-VIS 2001 spectrophotometer at 370nm absorbance (Divakaran et al., 2002). Apparent nutrient digestibility coefficient (ADC\%) for experimental diets was calculated by the standard formula (NRC, 1993) (Equation 4).

$$
A D C(\%)=100-100 \times \frac{\% \text { marker in diet } \times \% \text { nutrient in feces }}{\% \text { marker in feces } \times \% \text { nutrient in diet }}
$$

\subsection{Statistical analysis}

The individual and interactive effects of CA and PHY were calculated by applying three-way analysis of variance on obtained data. The results were considered significant at $\mathrm{p}<0.05$ (Snedecor and Cochran, 1991). The CoStat computer package (Version 6.303, PMB 320, Monterey, CA, 93940 USA) was used for statistical analysis.

\section{Results}

\subsection{Growth performance}

Table 2 shows the growth parameters of $C$. mrigala fingerlings fed on PHY and CA supplemented CM based diet. It was recorded that both the supplementations individually brought significant changes in improving growth performance of $C$. mrigala fingerlings. Dietary acidification through the inclusion of CA lowered the $\mathrm{pH}$ of the fish gut in dose dependent manner and improved the growth parameters $(\mathrm{p}<0.05)$. Phytate hydrolysis was maximum when both the supplements interacted synergistically at $\mathrm{T}_{12}$ diet; having $50 \%$ FM substituted with CM supplemented with 2.5\% CA and 750 FTU kg-1 level of phytase supplementation. Significantly $(\mathrm{p}<0.05)$ improved weight gain (WG) (18g), weight gain\% (WG\%) (288g), survival rate (100\%) and lowest FCR (1.07) was noted at this level which was different from the fish fed on control diet and all other supplemented levels as shown in Figures 1 and 2. Second best results were noted down in $\mathrm{T}_{11}$; in which $750 \mathrm{FTU} \mathrm{kg}^{-1} \mathrm{PHY}$ rendered the $\mathrm{WG} \%$ and FCR to $253 \%$ and 1.21 , respectively. The lowest values of growth indices such as WG (9g), WG\% (156\%), FCR (2.02) and survival (96\%) were observed in the fish fed on $\mathrm{T}_{1}$ test diet, having more inclusion of FM than the $\mathrm{CM}$ with $0 \% \mathrm{CA}$ and 0 FTU $\mathrm{kg}^{-1}$ level of PHY supplementation. The growth parameters started to increase positively with the increase in CM along with CA and PHY interactive level and then

\begin{tabular}{|c|c|c|c|c|c|c|c|c|c|c|c|}
\hline $\begin{array}{l}\text { Test } \\
\text { diets }\end{array}$ & $\begin{array}{c}\text { Fishmeal } \\
\text { Protein } \\
\text { Replacement } \\
\text { Levels (\%) } \\
\end{array}$ & Fishmeal & $\begin{array}{c}\text { Canola } \\
\text { meal }\end{array}$ & $\begin{array}{c}\text { PHY } \\
(\text { FTU kg-1) }\end{array}$ & $\begin{array}{l}\text { CA } \\
(\%)\end{array}$ & $\begin{array}{c}\text { Rice } \\
\text { polish }\end{array}$ & $\begin{array}{l}\text { Wheat } \\
\text { flour }\end{array}$ & $\begin{array}{c}\text { Fish } \\
\text { oil }\end{array}$ & $\begin{array}{l}\text { Vitamins } \\
\text { Premix }\end{array}$ & $\begin{array}{l}\text { Minerals } \\
\text { Premix }\end{array}$ & $\begin{array}{c}\text { Chromic } \\
\text { Oxide }\end{array}$ \\
\hline $\mathrm{T}_{1}$ & $0 \%$ & 48 & 18 & 0 & 0 & 3 & 22 & 6 & 1 & 1 & 1 \\
\hline $\mathrm{T}_{2}$ & & 48 & 18 & & 2.5 & 3 & 19.5 & 6 & 1 & 1 & 1 \\
\hline $\mathrm{T}_{3}^{2}$ & & 48 & 18 & 750 & 0 & 3 & 22 & 6 & 1 & 1 & 1 \\
\hline $\mathrm{T}_{4}^{3}$ & & 48 & 18 & & 2.5 & 3 & 19.5 & 6 & 1 & 1 & 1 \\
\hline $\mathrm{T}_{5}^{4}$ & $25 \%$ & 36 & 35 & 0 & 0 & 3 & 17 & 6 & 1 & 1 & 1 \\
\hline $\mathrm{T}_{6}^{5}$ & & 36 & 35 & & 2.5 & 3 & 14.5 & 6 & 1 & 1 & 1 \\
\hline $\mathrm{T}_{7}^{6}$ & & 36 & 35 & 750 & 0 & 3 & 17 & 6 & 1 & 1 & 1 \\
\hline $\mathrm{T}_{8}^{\prime}$ & & 36 & 35 & & 2.5 & 3 & $14.5 / 15$ & 6 & 1 & 1 & 1 \\
\hline $\mathrm{T}_{9}^{8}$ & $50 \%$ & 24 & 53 & 0 & 0 & 3 & 11 & 6 & 1 & 1 & 1 \\
\hline $\mathrm{T}_{10}^{\mathrm{g}}$ & & 24 & 53 & & 2.5 & 3 & 8.5 & 6 & 1 & 1 & 1 \\
\hline $\mathrm{T}_{11}^{10}$ & & 24 & 53 & 750 & 0 & 3 & 11 & 6 & 1 & 1 & 1 \\
\hline $\mathrm{T}_{12}$ & & 24 & 53 & & 2.5 & 3 & 8.5 & 6 & 1 & 1 & 1 \\
\hline $\mathrm{T}_{13}^{12}$ & $75 \%$ & 12 & 71 & 0 & 0 & 3 & 5 & 6 & 1 & 1 & 1 \\
\hline $\mathrm{T}_{14}^{13}$ & & 12 & 71 & & 2.5 & 3 & 2.5 & 6 & 1 & 1 & 1 \\
\hline $\mathrm{T}_{15}^{14}$ & & 12 & 71 & 750 & 0 & 3 & 5 & 6 & 1 & 1 & 1 \\
\hline $\mathrm{T}_{16}^{15}$ & & 12 & 71 & & 2.5 & 3 & 2.5 & 6 & 1 & 1 & 1 \\
\hline
\end{tabular}
changed inversely with the further increase in CM level.

Table 1. Ingredients composition (\%) of test diets. 
Table 2. Growth parameters of C. mrigala fingerlings fed on PHY and CA supplemented canola meal based test diets.

\begin{tabular}{|c|c|c|c|c|c|c|c|c|}
\hline $\begin{array}{l}\text { Test } \\
\text { diets }\end{array}$ & $\begin{array}{c}\text { Fishmeal } \\
\text { Protein } \\
\text { Replacement } \\
\text { Levels (\%) } \\
\end{array}$ & $\begin{array}{c}\text { PHY } \\
(\text { FTU kg-1) }\end{array}$ & CA (\%) & Initial weight & Final weight & Weight gain & SGR & Survival\% \\
\hline $\mathrm{T}_{1}$ & $0 \%$ & 0 & 0 & $6.35 \pm 0.040$ & $16.30 \pm 0.654^{\mathrm{j}}$ & $9.95 \pm 0.618^{\mathrm{i}}$ & $1.04 \pm 0.038^{\mathrm{b}}$ & 96 \\
\hline $\mathrm{T}_{2}$ & & & 2.5 & $6.500 \pm 0.026$ & $17.813 \pm 0.428^{\mathrm{i}}$ & $11.313 \pm 0.450^{\mathrm{h}}$ & $1.11 \pm 0.030^{\mathrm{b}}$ & 96 \\
\hline $\mathrm{T}_{3}^{2}$ & & 750 & 0 & $6.570 \pm 0.040$ & $18.743 \pm 0.585^{\mathrm{h}}$ & $12.173 \pm 0.613^{g}$ & $1.16 \pm 0.039^{\mathrm{b}}$ & 98 \\
\hline $\mathrm{T}_{4}^{3}$ & & & 2.5 & $6.510 \pm 0.040$ & $21.023 \pm 0.912 \mathrm{f}$ & $14.513 \pm 0.899^{\mathrm{e}}$ & $1.30 \pm 0.045^{\mathrm{b}}$ & 98 \\
\hline $\mathrm{T}_{5}^{4}$ & $25 \%$ & 0 & 0 & $6.543 \pm 0.042$ & $17.853 \pm 0.465^{\mathrm{i}}$ & $11.310 \pm 0.426^{\mathrm{h}}$ & $1.11 \pm 0.022^{\mathrm{b}}$ & 96 \\
\hline $\mathrm{T}_{6}^{3}$ & & & 2.5 & $6.557 \pm 0.122$ & $20.230 \pm 0.969^{g}$ & $13.673 \pm 1.084^{\mathrm{f}}$ & $1.25 \pm 0.072^{\mathrm{b}}$ & 98 \\
\hline $\mathrm{T}_{7}^{6}$ & & 750 & 0 & $6.570 \pm 0.053$ & $21.150 \pm 0.350^{\mathrm{f}}$ & $14.580 \pm 0.367^{\mathrm{e}}$ & $1.29 \pm 0.022^{\mathrm{b}}$ & 98 \\
\hline $\mathrm{T}_{8}^{\prime}$ & & & 2.5 & $6.597 \pm 0.101$ & $22.223 \pm 0.665^{\mathrm{d}}$ & $15.627 \pm 0.565^{d}$ & $1.34 \pm 0.016^{\mathrm{b}}$ & 100 \\
\hline $\mathrm{T}_{9}^{8}$ & $50 \%$ & 0 & 0 & $6.523 \pm 0.091$ & $18.670 \pm 0.901^{\mathrm{h}}$ & $12.147 \pm 0.986^{g}$ & $1.16 \pm 0.068^{\mathrm{b}}$ & 96 \\
\hline $\mathrm{T}_{10}^{\mathrm{g}}$ & & & 2.5 & $6.570 \pm 0.050$ & $19.930 \pm 0.527^{\mathrm{g}}$ & $13.360 \pm 0.490^{\mathrm{f}}$ & $1.23 \pm 0.023^{\mathrm{b}}$ & 98 \\
\hline $\mathrm{T}_{11}^{10}$ & & 750 & 0 & $6.563 \pm 0.092$ & $23.397 \pm 1.181^{b}$ & $16.787 \pm 1.110^{\mathrm{b}}$ & $1.40 \pm 0.045^{\mathrm{b}}$ & 100 \\
\hline $\mathrm{T}_{12}$ & & & 2.5 & $0.040 \pm 0.040$ & $25.403 \pm 0.344^{\mathrm{a}}$ & $18.870 \pm 0.310^{\mathrm{a}}$ & $1.50 \pm 0.009^{a}$ & 100 \\
\hline $\mathrm{T}_{12}^{12}$ & $75 \%$ & 0 & 0 & $6.580 \pm 0.082$ & $19.737 \pm 0.558^{\mathrm{g}}$ & $13.157 \pm 0.600^{f}$ & $1.22 \pm 0.039^{\mathrm{b}}$ & 96 \\
\hline $\mathrm{T}_{14}^{13}$ & & & 2.5 & $6.660 \pm 0.050$ & $21.590 \pm 0.598^{e}$ & $14.930 \pm 0.598^{e}$ & $1.30 \pm 0.031^{\mathrm{b}}$ & 100 \\
\hline $\mathrm{T}_{15}^{14}$ & & 750 & 0 & $6.563 \pm 0.076$ & $21.690 \pm 0.486^{e}$ & $15.127 \pm 0.478^{e}$ & $1.32 \pm 0.025^{\mathrm{b}}$ & 100 \\
\hline $\mathrm{T}_{16}^{\mathrm{IS}}$ & & & 2.5 & $6.577 \pm 0.049$ & $22.810 \pm 0.688^{c}$ & $16.233 \pm 0.653^{c}$ & $1.38 \pm 0.027^{\mathrm{b}}$ & 100 \\
\hline
\end{tabular}

Means within columns having different superscripts are significantly different at $\mathrm{p}<0.05$. Data are means of three replicates. SGR; Standard Growth Rate.

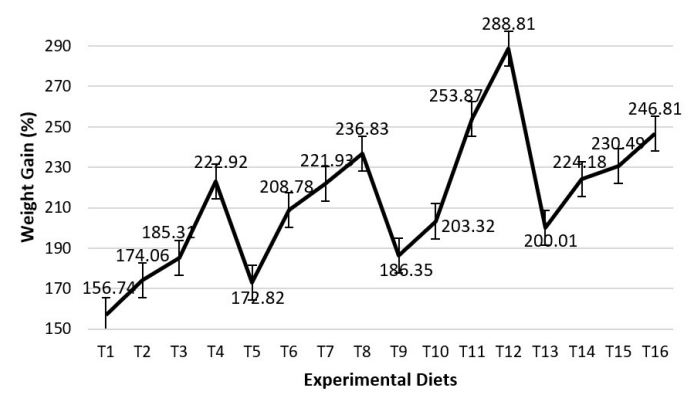

Figure 1. Weight gain (\%) of $C$. mrigala fingerlings fed on CM based diets supplemented with PHY and CA.

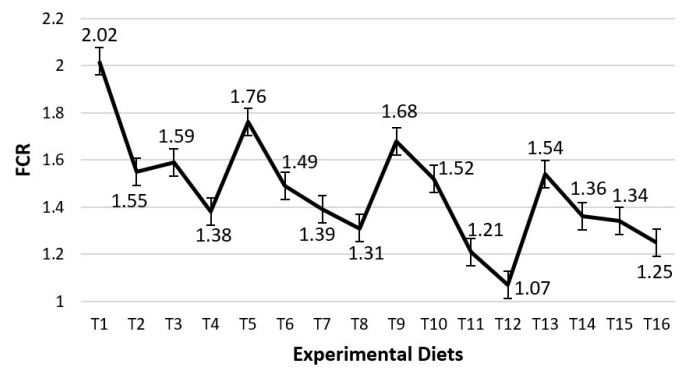

Figure 2. FCR of $C$. mrigala fingerlings fed on $\mathrm{CM}$ based diets supplemented with PHY and CA.

\subsection{Apparent nutrient digestibility}

the nutrients percentage in feed and feces of $C$. mrigala fingerlings and its digestibility values are given in Tables 3, 4 and 5, respectively. Notably highest ADC (\%) (CP: 77\%, CF: 84\% and GE: 70\%) were observed in the fish group fed test diet $\mathrm{T}_{12}$ while the lowest values of nutrients digestibility (CP: 51\%, CF: $65 \%$ and GE: 53\%) were observed in the fish fed with control test diet $\left(\mathrm{T}_{1}\right)$ showing that simultaneous supplementation of phytase and citric acid ( $\mathrm{CA} \times \mathrm{PHY})$ in canola meal based diets enhanced the nutrient digestibility by degrading the phytate complexes. By breakdown of phytate, the essential nutrients were released and became available for the fish to fulfill its nutritional demands. Individual role of phytase at 750 FTUkg-1 $^{-1}$ was also significant $\left(\mathrm{T}_{11}\right)$ and brought $\mathrm{CP}, \mathrm{CF}$, and GE to $76 \%, 83 \%$ and $68 \%$, respectively. Results of present work also suggested that high dose of phytase level i.e., $750 \mathrm{FTU} \mathrm{kg}^{-1}$ when combined with optimum level of CA (2.5\%), resulted in improved nutrient digestibility (Table 5). Due to increased nutrient digestibility of diet, growth performance was also enhanced. So, highly significant $(p<0.05)$ differences in digestibility of nutrients of the $\left(T_{1}\right)$ and that of the treated levels can be observed. Hence, it can be deduced from the above mentioned results that nutrient digestibility parameters were improved when both the supplements (CA×PHY) interacted synergistically with increasing CM levels but up to $50 \%$, afterwards it showed inverse relationship.

\section{Discussion}

When CA is used in combination with PHY in plant meal based fish diet, the release of $\mathrm{H}^{+}$ions show positive results by reducing the $\mathrm{pH}$ of the stomach, breaking down the PHY complexes and intensifying the PHY activity (Shah et al., 2015, 2021). In the current study, the maximum values of growth performance for $C$. mrigala fingerlings were noted at $2.5 \% \mathrm{CA}$ and $750\left(\mathrm{FTU} \mathrm{kg}^{-1}\right)$ PHY supplementation in diets having 50\% FM substitution level. Likewise, Mahmoud et al. (2019) and Nehad et al. (2020) found significantly improved $(\mathrm{p}<0.05)$ growth performance (WG\%: 67\%, FCR: 1.50) and feed utilization parameters while studying interactive effects of CA and PHY at $30 \mathrm{~g} / \mathrm{kg}$ and 1000 FTUkg $^{-1}$, respectively in Oreochromis niloticus. This is in agreement with Baruah et al. (2007), who found maximum growth performance at $3 \% \mathrm{CA}$ and $500\left(\mathrm{FTU} \mathrm{kg}^{-1}\right)$ 
Table 3. Analyzed compositions (\%) of apparent crude protein, crude fat and gross energy in the diets of C. mrigala fingerlings fed on PHY and CA supplemented canola meal based test diets.

\begin{tabular}{|c|c|c|c|c|c|c|}
\hline $\begin{array}{c}\text { Test } \\
\text { Diets }\end{array}$ & $\begin{array}{c}\text { Fishmeal Protein } \\
\text { Replacement } \\
\text { Levels (\%) }\end{array}$ & $\begin{array}{c}\text { PHY } \\
\left(\text { FTU kg } \text { kg-1) }^{-1}\right.\end{array}$ & CA (\%) & Crude protein & Crude fat & Gross energy \\
\hline $\mathrm{T}_{1}$ & $0 \%$ & 0 & 0 & $30.54 \pm 0.066$ & $8.05 \pm 0.020$ & $3.41 \pm 0.020$ \\
\hline $\mathrm{T}_{2}$ & & & 2.5 & $30.62 \pm 0.090$ & $8.08 \pm 0.040$ & $3.43 \pm 0.030$ \\
\hline $\mathrm{T}_{3}$ & & 750 & 0 & $30.58 \pm 0.035$ & $8.05 \pm 0.030$ & $3.4 \pm 0.017$ \\
\hline $\mathrm{T}_{4}$ & & & 2.5 & $30.65 \pm 0.079$ & $8.05 \pm 0.030$ & $3.42 \pm 0.025$ \\
\hline $\mathrm{T}_{5}$ & $25 \%$ & 0 & 0 & $30.64 \pm 0.050$ & $8.07 \pm 0.020$ & $3.41 \pm 0.036$ \\
\hline $\mathrm{T}_{6}$ & & & 2.5 & $30.63 \pm 0.090$ & $8.04 \pm 0.020$ & $3.43 \pm 0.017$ \\
\hline $\mathrm{T}_{7}$ & & 750 & 0 & $30.66 \pm 0.049$ & $8.08 \pm 0.015$ & $3.41 \pm 0.025$ \\
\hline $\mathrm{T}_{8}$ & & & 2.5 & $30.66 \pm 0.040$ & $8.07 \pm 0.040$ & $3.42 \pm 0.026$ \\
\hline $\mathrm{T}_{9}$ & $50 \%$ & 0 & 0 & $30.65 \pm 0.077$ & $8.05 \pm 0.030$ & $3.42 \pm 0.030$ \\
\hline $\mathrm{T}_{10}$ & & & 2.5 & $30.66 \pm 0.062$ & $8.03 \pm 0.015$ & $3.42 \pm 0.02$ \\
\hline $\mathrm{T}_{11}$ & & 750 & 0 & $30.62 \pm 0.03$ & $8.04 \pm 0.010$ & $3.41 \pm 0.02$ \\
\hline $\mathrm{T}_{12}$ & & & 2.5 & $30.67 \pm 0.060$ & $8.06 \pm 0.010$ & $3.41 \pm 0.015$ \\
\hline $\mathrm{T}_{13}$ & $75 \%$ & 0 & 0 & $30.68 \pm 0.045$ & $8.05 \pm 0.020$ & $3.41 \pm 0.010$ \\
\hline $\mathrm{T}_{14}$ & & & 2.5 & $30.65 \pm 0.080$ & $8.08 \pm 0.040$ & $3.43 \pm 0.020$ \\
\hline $\mathrm{T}_{15}$ & & 750 & 0 & $30.69 \pm 0.020$ & $8.04 \pm 0.010$ & $3.42 \pm 0.025$ \\
\hline $\mathrm{T}_{16}$ & & & 2.5 & $30.63 \pm 0.041$ & $8.03 \pm 0.010$ & $3.42 \pm 0.04$ \\
\hline
\end{tabular}

Data are means of three replicates.

Table 4. Analyzed compositions (\%) of apparent crude protein, crude fat and gross energy in feces of $C$. mrigala fingerlings fed on PHY and CA supplemented canola meal based test diets.

\begin{tabular}{|c|c|c|c|c|c|c|}
\hline $\begin{array}{c}\text { Test } \\
\text { Diets }\end{array}$ & $\begin{array}{c}\text { Fishmeal } \\
\text { Protein } \\
\text { Replacement } \\
\text { Levels (\%) }\end{array}$ & $\begin{array}{c}\text { PHY } \\
(\text { FTU kg-1) }\end{array}$ & CA (\%) & Crude protein & Crude fat & Gross energy \\
\hline $\mathrm{T}_{1}$ & $0 \%$ & 0 & 0 & $15.72 \pm 0.448^{\mathrm{a}}$ & $2.24 \pm 0.025^{\mathrm{a}}$ & $1.69 \pm 0.015^{\mathrm{a}}$ \\
\hline $\mathrm{T}_{2}$ & & & 2.5 & $14.01 \pm 0.065^{c}$ & $2.01 \pm 0.036^{c}$ & $1.6 \pm 0.01^{c}$ \\
\hline $\mathrm{T}_{3}$ & & 750 & 0 & $13.34 \pm 0.0361^{\mathrm{d}}$ & $1.94 \pm 0.032^{\mathrm{d}}$ & $1.58 \pm 0.01^{\mathrm{d}}$ \\
\hline $\mathrm{T}_{4}$ & & & 2.5 & $10.55 \pm 0.025^{j}$ & $1.40 \pm 0.015^{j}$ & $1.39 \pm 0.015^{j}$ \\
\hline $\mathrm{T}_{5}$ & $25 \%$ & 0 & 0 & $14.68 \pm 0.065^{\mathrm{b}}$ & $2.1 \pm 0.02^{\mathrm{b}}$ & $1.63 \pm 0.011^{\mathrm{b}}$ \\
\hline $\mathrm{T}_{6}$ & & & 2.5 & $11.36 \pm 0.061^{\mathrm{h}}$ & $1.52 \pm 0.025^{\mathrm{h}}$ & $1.46 \pm 0.015^{\mathrm{h}}$ \\
\hline $\mathrm{T}_{7}$ & & 750 & 0 & $10.95 \pm 0.047^{\mathrm{i}}$ & $1.47 \pm 0.015^{\mathrm{i}}$ & $1.42 \pm 0.015^{\mathrm{i}}$ \\
\hline $\mathrm{T}_{8}$ & & & 2.5 & $8.92 \pm 0.035^{\mathrm{m}}$ & $1.76 \pm 0.036^{e}$ & $1.30 \pm 0.011^{\mathrm{m}}$ \\
\hline $\mathrm{T}_{9}$ & $50 \%$ & 0 & 0 & $12.92 \pm 0.055^{\mathrm{e}}$ & $1.75 \pm 0.03^{\mathrm{e}}$ & $1.56 \pm 0.026^{\mathrm{e}}$ \\
\hline $\mathrm{T}_{10}$ & & & 2.5 & $11.75 \pm 0.03^{\mathrm{g}}$ & $1.57 \pm 0.026^{g}$ & $1.49 \pm 0.02^{g}$ \\
\hline $\mathrm{T}_{11}$ & & 750 & 0 & $7.96 \pm 0.0378^{\circ}$ & $1.08 \pm 0.015^{\mathrm{m}}$ & $1.19 \pm 0.015^{\circ}$ \\
\hline $\mathrm{T}_{12}$ & & & 2.5 & $7.7 \pm 0.075^{p}$ & $1.02 \pm 0.01^{\mathrm{n}}$ & $1.12 \pm 0.025^{p}$ \\
\hline $\mathrm{T}_{13}$ & $75 \%$ & 0 & 0 & $12.14 \pm 0.050^{\mathrm{f}}$ & $1.64 \pm 0.015^{f}$ & $1.53 \pm 0.015^{\mathrm{f}}$ \\
\hline $\mathrm{T}_{14}$ & & & 2.5 & $10.03 \pm 0.06 \mathrm{k}$ & $1.76 \pm 0.036^{e}$ & $1.36 \pm 0.02^{\mathrm{k}}$ \\
\hline $\mathrm{T}_{15}$ & & 750 & 0 & $9.43 \pm 0.035^{1}$ & $1.28 \pm 0.032^{\mathrm{k}}$ & $1.33 \pm 0.015^{1}$ \\
\hline $\mathrm{T}_{16}$ & & & 2.5 & $8.21 \pm 0.035^{\mathrm{n}}$ & $1.14 \pm 0.02^{1}$ & $1.26 \pm 0.015^{\mathrm{n}}$ \\
\hline
\end{tabular}

Means within columns having different superscripts are significantly different at $\mathrm{p}<0.05$. Data are means of three replicates.

PHY supplementation level. Similarly, Zhu et al. (2014) and Thiam et al. (2015) reported that the interaction of CA with PHY worked best to improve growth and protein content of yellow catfish and Nile tilapia fingerlings, respectively. A recent study conducted by Fadhal and Mustafa (2020) confirmed the role of phytase as a growth promoting dietary additive when used at $4000 \mathrm{IU} / \mathrm{kg}$ in the diet of common carp. Similar results of optimized growth were reported by Hussain et al. (2015) but the levels were different from our study. They recorded that supplementation of 5\% CA and 500 (FTU kg-1) PHY resulted in the maximum growth (WG\%: 401\% and FCR: 1.21 ) of C. mrigala fingerlings fed canola meal-based diet. In recent researches, it was found that incorporation of CA and PHY in fish feed must be around 2.5-5\% and 250-1500 FTUkg-1 (Cao et al., 2007; Khajepour et al., 2012). 
Table 5. Apparent Digestibility Coefficient (ADC\%) of crude protein, crude fat and gross energy of C. mrigala fingerlings fed on PHY and CA supplemented canola meal based test diets.

\begin{tabular}{|c|c|c|c|c|c|c|}
\hline $\begin{array}{c}\text { Test } \\
\text { Diets }\end{array}$ & $\begin{array}{c}\text { Fishmeal Protein } \\
\text { Replacement } \\
\text { Levels (\%) }\end{array}$ & $\begin{array}{c}\text { PHY } \\
\left(\text { FTU kg }^{-1}\right)\end{array}$ & CA (\%) & Crude protein & Crude fat & Gross energy \\
\hline $\mathrm{T}_{1}$ & $0 \%$ & 0 & 0 & $51.68 \pm 0.522^{\circ}$ & $65.25 \pm 0.488^{\circ}$ & $53.51 \pm 0.948^{p}$ \\
\hline $\mathrm{T}_{2}$ & & & 2.5 & $54.85 \pm 2.383^{n}$ & $49.30 \pm 10.047^{p}$ & $55.97 \pm 1.018^{\mathrm{n}}$ \\
\hline $\mathrm{T}_{3}$ & & 750 & 0 & $59.28 \pm 0.654^{\mathrm{m}}$ & $70.16 \pm 0.842^{\mathrm{m}}$ & $56.63 \pm 0.665^{\mathrm{m}}$ \\
\hline $\mathrm{T}_{4}$ & & & 2.5 & $67.47 \pm 0.566^{g}$ & $78.22 \pm 0.209^{g}$ & $61.72 \pm 0.686^{g}$ \\
\hline $\mathrm{T}_{5}$ & $25 \%$ & 0 & 0 & $54.73 \pm 0.398^{n}$ & $67.29 \pm 0.203^{n}$ & $55.01 \pm 0.307^{\circ}$ \\
\hline $\mathrm{T}_{6}$ & & & 2.5 & $65.47 \pm 1.084^{\mathrm{i}}$ & $76.66 \pm 0.412^{\mathrm{i}}$ & $60.45 \pm 1.709^{i}$ \\
\hline $\mathrm{T}_{7}$ & & 750 & 0 & $66.45 \pm 0.862^{\mathrm{h}}$ & $77.37 \pm 0.346^{\mathrm{h}}$ & $60.91 \pm 1.694^{\mathrm{h}}$ \\
\hline $\mathrm{T}_{8}$ & & & 2.5 & $73.39 \pm 0.505^{\mathrm{d}}$ & $81.07 \pm 0.259^{\mathrm{d}}$ & $65.33 \pm 0.287^{\mathrm{d}}$ \\
\hline $\mathrm{T}_{9}$ & $50 \%$ & 0 & 0 & $61.22 \pm 1.328^{1}$ & $73.39 \pm 0.452^{1}$ & $57.99 \pm 1.755^{1}$ \\
\hline $\mathrm{T}_{10}$ & & & 2.5 & $64.47 \pm 0.369^{j}$ & $75.62 \pm 0.136^{j}$ & $59.61 \pm 0.839^{j}$ \\
\hline $\mathrm{T}_{11}$ & & 750 & 0 & $76.61 \pm 0.314^{\mathrm{b}}$ & $83.97 \pm 0.009^{b}$ & $68.74 \pm 0.414^{\mathrm{b}}$ \\
\hline $\mathrm{T}_{12}$ & & & 2.5 & $77.38 \pm 0.340^{\mathrm{a}}$ & $84.81 \pm 0.063^{a}$ & $70.47 \pm 0.400^{a}$ \\
\hline $\mathrm{T}_{13}$ & $75 \%$ & 0 & 0 & $63.05 \pm 0.884^{\mathrm{k}}$ & $74.71 \pm 0.602^{\mathrm{k}}$ & $58.16 \pm 0.829^{k}$ \\
\hline $\mathrm{T}_{14}$ & & & 2.5 & $69.73 \pm 0.315^{f}$ & $79.50 \pm 0.193^{f}$ & $63.29 \pm 0.130^{\mathrm{f}}$ \\
\hline $\mathrm{T}_{15}$ & & 750 & 0 & $71.58 \pm 0.249^{e}$ & $80.41 \pm 0.408^{e}$ & $64.12 \pm 0.505^{\mathrm{e}}$ \\
\hline $\mathrm{T}_{16}$ & & & 2.5 & $75.63 \pm 0.358^{c}$ & $82.79 \pm 0.167^{c}$ & $66.68 \pm 0.116^{c}$ \\
\hline
\end{tabular}

Means within columns having different superscripts are significantly different at $\mathrm{p}<0.05$. Data are means of three replicates.

Data in the present study proved that a significant difference exist in the nutrient digestibility of crude protein, crude fat and gross energy at both individual and interactive levels of CA and PHY supplemented CM based diets. The findings support the hypothesis that addition of organic acid in the diet gives the favorable environment to PHY for optimum performance. Shah et al. (2021) recorded the improved nutrient utilization and hence improved growth of $L$. rohita fingerlings by using combined effect of PHY and CA at 1000 FTUkg $^{-1}$ and $30 \mathrm{~g} / \mathrm{kg}$ in sunflower meal based diets. Both of these supplements show synergistic effect of phytate hydrolysis when used in combination. This difference in concentration can be due to varied experimental conditions and test species. $20 \mathrm{~g} / \mathrm{kg}$ optimum supplementation level. To conclude, the supplementation of this organic acid in diets does not negatively affect fish nutritional status, health or welfare, and increases nutrient and mineral availability. Nascimento et al. (2021) also found increased ADC (\%) for crude energy and dry matter when fed Colossoma macropomum on plant based diet supplemented with $18.5 \mathrm{~g} / \mathrm{kg}$ citric acid. Similar to our results, positive results of growth performance and feed utilization were found by different researchers on a number of species including C. mrigala (Hussain et al., 2017), tilapia (Maas et al., 2018) and L. rohita (Bano and Afzal, 2018).

Results of the present study are also supported by Hussain et al. (2011) who stated that 750 (FTU kg-1) PHY supplementation level with plant-meal based diet is optimal for highest growth performance of $L$. rohita fingerlings. In contrast to our study, Khajepour and
Hosseini (2012) reported that the addition of citric acid in the fish diet reduced the digestibility of lipid because he claimed a decrease in fat digestibility with PHY addition. Similarly, contrasting results of non-significant change in growth rate, feed intake or feed efficiency was recorded by Dai et al. (2018) in turbot. In the same way, Yigit et al. (2018) also reported the non-significant effect of phytase supplementation in soybean meal based diets even at $2 \mathrm{~g} / \mathrm{kg}$ level, resultantly growth parameters and feed utilization parameters remain unaffected. Its possible reason can be different dietary factors such as sources and concentration of phytase in the diet, type of feed ingredients, methods of fish drying, age and size of fish and fish species.

\section{Conclusion}

Under the experimental conditions tested herein, it can be said that addition of CA and PHY in CM based diets enhanced digestibility of nutrients for $C$. mrigala fingerlings, which in turn led to the improved growth performance. It was also deduced that test diet $\mathrm{T}_{12}$ having $50 \%$ fishmeal substituted with canola meal supplemented with $2.5 \%$ CA and 750 FTU kg-1 PHY level proved to be the optimum diet for the improvement of the all above said factors. Acidification of PHY with CA proved to be efficient in formulating nutritionally bioavailable, cost effective and eco-friendly feed for C. mrigala. Besides, there is need of detailed study on different size and age group of fish. 


\section{Acknowledgements}

The authors would like to acknowledge HEC Pakistan for providing funds for Project \# 20-4892/NRPU/R\&D/ HEC/14/1145 to conduct this research work.

\section{References}

ABID, M. and AHMED, M.S., 2009. Growth response of Labeo rohita fingerlings fed with different feeding regimes under intensive rearing. Journal of Animal and Plant Science, vol. 19, no. 1, pp. 45-49.

ALHAZZAA, R., NICHOLS, P.D. and CARTER, C.G., 2019. Sustainable alternatives to dietary fish oil in tropical fish aquaculture. Reviews in Aquaculture, vol. 11, no. 4, pp. 1195-1218. http:// dx.doi.org/10.1111/raq.12287.

ALLAN, G.L. and ROWLAND, S.J., 1992. Development of an experimental diet for silver perch (Bidyanus bidyanus). Austasia Aquaculture, vol. 6, pp. 39-40.

ASSOCIATION OF OFFICIAL ANALYTICAL CHEMISTS - AOAC, 2005. Official methods of analysis. 15th ed. Washington: AOAC, $1094 \mathrm{p}$.

BANO, N. and AFZAL, M., 2018. Synchronized effect of citric acid and phytase supplementation on growth performance and nutrient digestibility of Labeo rohita. Aquaculture Nutrition, vol. 24, no. 2, pp. 786-792. http://dx.doi.org/10.1111/anu.12607.

BARUAH, K., SAHU, N.P., PAL, A.K., JAIN, K.K., DEBNATH, D. and MUKHERJEE, S.C., 2007. Dietary microbial phytase and citric acid synergistically enhances nutrient digestibility and growth performance of Labeo rohita (Hamilton) juveniles at sub-optimal protein level. Aquaculture Research, vol. 38, no. 2, pp. 109-120. http://dx.doi.org/10.1111/j.1365-2109.2006.01624.x.

CAO, L., WANG, W., YANG, C., YANG, Y., DIANA, J., YAKUPITIYAGE, A., LUO, Z. and LI, D., 2007. Application of microbial phytase in fish feed. Enzyme and Microbial Technology, vol. 40, no. 4, pp. 497-507. http://dx.doi.org/10.1016/j.enzmictec.2007.01.007.

CHENG, Z.J. and HARDY, R.W., 2002. Effect of microbial phytase on apparent nutrient digestibility of barley, canola meal, wheat and wheat middlings, measured in vivo using rainbow trout (Oncorhynchus mykiss). Aquaculture Nutrition, vol. 8, no. 4, pp. 271-277. http://dx.doi.org/10.1046/j.1365-2095.2002.00219.x.

DAI, J., LI, Y., YANG, P., LIU, Y., CHEN, Z., OU, W., AI, Q., ZHANG, W., ZHANG, Y. and MAI, K., 2018. Citric acid as a functional supplement in diets for juvenile turbot, Scophthalmus maximus L.: effects on phosphorus discharge, growth performance, and intestinal health. Aquaculture, vol. 495, pp. 643-653. http:// dx.doi.org/10.1016/j.aquaculture.2018.04.004.

DIVAKARAN, S., OBALDO, L.G. and FORSTER, I.P., 2002. Note on the methods for determination of chromic oxide in shrimp feeds. Journal of Agricultural and Food Chemistry, vol. 50, no. 3, pp. 464-467. http://dx.doi.org/10.1021/jf011112s. PMid:11804513.

FADHAL, A.A. and MUSTAFA, S.A., 2020. Influence of phytase enzyme on growth performance and survival rate challenged with Saprolegnia spp. in common carp. The Iraqi Journal of Agricultural Science, vol. 51, no. 5, pp. 1458-1465. http://dx.doi. org/10.36103/ijas.v51i5.1156.

GLENCROSS, B., 2016. Understanding the nutritional and biological constraints of ingredients to optimize their application in aquaculture feeds. In: S.F. NATES, ed. Aquafeed formulation. New York: Academic Press, pp. 32-73. http://dx.doi.org/10.1016/ B978-0-12-800873-7.00003-8.

HUSSAIN, S., AFZAL, M., RANA, S.A., JAVID, A. and IQBAL, M., 2011 [viewed 14 December 2020]. Effect of phytase supplementation on growth performance and nutrient digestibility of Labeo rohita fingerlings fed on corn gluten meal-based diets. International Journal of Agriculture and Biology [online], vol. 13, no. 6, pp. 916-922. Available from: http://www.fspublishers.org

HUSSAIN, S.M., HAMEED, T., AFZAL, M., JAVID, A., ASLAM, N., SHAH, S.Z.H., HUSSAIN, M., ARSALAN, M.Z.H. and SHAHZAD, M.M., 2017. Growth performance and nutrient digestibility of Cirrhinus mrigala fingerlings fed phytase supplemented sunflower meal based diet. Pakistan Journal of Zoology, vol. 49, no. 5, pp. 1713-1724. http://dx.doi.org/10.17582/journal. pjz/2017.49.5.1713.1724.

HUSSAIN, S.M., NISAR, A., FARHAT, J., ARSHAD, J., NOSHEEN, A., MAJID, H., SHAHTAJ, A., ARSALAN, M.Z.H., DANISH, R. and SHAHZAD, M.M., 2015. Effects of citric acid and phytase supplementation on nutrient digestibility and growth performance of Cirrhinus mrigala fingerlings fed on corn gluten (30\%) meal based diets. International Journal of Biosciences, vol. 6, no. 7, pp. 82-91. http://dx.doi.org/10.12692/ijb/6.7.82-91.

JACKSON, L.S., LI, M.H. and ROBINSON, E.H., 1996. Use of microbial phytase in channel catfish Ictalurus punctatus diets to improve utilization of phytate phosphorus. Journal of the World Aquaculture Society, vol. 27, no. 3, pp. 309-313. http://dx.doi. org/10.1111/j.1749-7345.1996.tb00613.x.

KHAJEPOUR, F. and HOSSEINI, S.A., 2012. Citric acid improves growth performance and phosphorus digestibility in Beluga (Huso huso) fed diets where soybean meal partly replaced fishmeal. Animal Feed Science and Technology, vol. 171, no. 1, pp. 68-73. http://dx.doi.org/10.1016/j.anifeedsci.2011.10.001.

KHAJEPOUR, F., HOSSEINI, S.A. and IMANPOUR, M.R., 2012. Dietary crude protein, citric acid and microbial phytase and their interaction to influence growth performance, muscle proximate composition and hematocrite of common carp, Cyprinus carpio L, juveniles. World Journal of Zoology, vol. 7, no. 2, pp. 118-122.

KHAN, M.A., JAFRI, A.K. and CHADHA, N.K., 2004. Growth, reproductive performance, muscle and egg composition in grass carp, Ctenopharyngodon idella (Valenciennes), fed hydrilla or formulated diets with varying protein levels. Aquaculture Research, vol. 35, no. 13, pp. 1277-1285. http:// dx.doi.org/10.1111/j.1365-2109.2004.01150.x.

LIM, S.J. and LEE, K.J., 2009. Partial replacement of fishmeal by cottonseed meal and soybean meal with iron and phytase supplementation for parrot fish Oplegnathus fasciatus. Aquaculture, vol. 290, no. 3-4, pp. 283-289. http://dx.doi. org/10.1016/j.aquaculture.2009.02.018.

LOVELL, T., 1989. Nutrition and feeding of fish. New York: Van Nostrand Reinhold, vol. 260. http://dx.doi.org/10.1007/9781-4757-1174-5.

MAAS, R.M., VERDEGEM, M.C., DERSJANT-LI, Y. and SCHRAMA, J.W., 2018. The effect of phytase, xylanase and their combination on growth performance and nutrient utilization in Nile tilapia. Aquaculture, vol. 487, pp. 7-14. http://dx.doi.org/10.1016/j. aquaculture.2017.12.040.

MAHMOUD, N., EID, A., WAHDAN, A.A., ENANY, M.E., EL-NAB, A. and ASMAA, S., 2019. Effect of phytase and citric acid on growth performance, feed utilization and its antibacterial activity against fish pathogens of Nile tilapia fingerlings. Egyptian Journal for Aquaculture, vol. 9, no. 4, pp. 35-53. http://dx.doi. org/10.21608/eja.2019.47194.

MANUEL, A.V., SHAHABUDDIN, A.M., SAHA, D., CHELE, M.H., DA CONCEICAO, K.O., TSUTSUI, N. and YOSHIMATSU, T., 2019. Growth and feed utilization of juvenile Nile tilapia fed with boiled Moringa oleifera meal diets: a preliminary report. Journal of International Cooperation for Agricultural Development, vol. 17, pp. 8-13. 
MUSHTAQ, T., SARWAR, M., AHMAD, G., NISA, M.U. and JAMIL, A., 2006. The influence of exogenous multienzyme preparation and graded levels of digestible lysine in sunflower meal-based diets on the performance of young broiler chicks two weeks post-hatching. Poultry Science, vol. 85, no. 12, pp. 2180-2185. http://dx.doi.org/10.1093/ps/85.12.2180. PMid:17135675.

NASCIMENTO, M.D.S., MATTOS, B.O., BUSSONS, M.R., OLIVEIRA, A.T., LIEBL, A.R.D.S. and CARVALHO, T.B., 2021. Supplementation of citric acid in plant protein-based diets for juvenile tambaqui, Colossoma macropomum. Journal of the World Aquaculture Society, vol. 52, no. 1, pp. 231-243. http://dx.doi.org/10.1111/jwas.12735.

NATIONAL RESEARCH COUNCIL - NRC, 1993. Nutrient requirements of fish. Washington: National Academies Press, pp. 114.

NEHAD, M., EID, A.E., ALI, B.A., WAHDAN, A., ENANY, M.E. and ABD EL-NABY, A.S., 2020. Effect of cinnamaldehyde and yeast on growth performance, feed utilization and its antibacterial activity against fish pathogens of Nile tilapia fingerlings. Aquaculture, vol. 13, no. 1, pp. 19-42.

OLUSOLA, S.E. and NWANNA, L.C., 2014. Growth performance of Nile tilapia (Oreochromis niloticus) fed processed soybean meal based diets supplemented with phytase. International Journal of Aquaculture, vol. 4, pp. 48-54. http://dx.doi.org/10.5376/ ija.2014.04.0008.

PANDEY, A. and SATOH, S., 2014. Effects of organic acids on growth and phosphorus utilization in rainbow trout, Oncorhynchus mykiss. Journal of Environmental Biology, vol. 35, no. 6, pp. 1081-1085. PMid:25522509.

PAPATRYPHON, E., HOWELL, R.A. and SOARES, J.H., 1999. Growth and mineral absorption by striped bass Morone saxatilis fed a plant feedstuff based diet supplemented with phytase. Journal of the World Aquaculture Society, vol. 30, no. 2, pp. 161-173. http://dx.doi.org/10.1111/j.1749-7345.1999.tb00863.x.

PHROMKUNTHONG, W., NUNTAPONG, N. and GABAUDAN, J., 2010 [viewed 14 December 2020]. Interaction of phytase Ronozyme and citric acid on the utilization of phosphorus by common carp (Cyprinus carpio). Songklanakarin Journal of Science and Technology [online], vol. 32, no. 6, pp. 547-554. Available from: http://www.sjst.psu.ac.th

ROWLAND, S.J. and INGRAM, B.A., 1991. Diseases of Australian native fishes. Sydney: NSW Agriculture. Fish Bulletin, no. 4.
SALIN, K.R., ARUN, V.V., MOHANAKUMARAN NAIR, C. and TIDWELL, J.H., 2018. Sustainable aquafeed. In: F.I. HAI, C. VISVANATHAN and R. BOOPATHY, eds. Sustainable aquaculture. Cham: Springer, pp. 123-151. http://dx.doi.org/10.1007/978-3-319-73257-2_4.

SHAFIQUE, L., AFZAL, M., SHAH, S.Z.H., FATIMA, M., NAZ, H. and SADDIQUE, Q., 2018. Acidified diet increases the trace mineral content in whole body fish Labeo rohita fingerlings. Punjab University Journal of Zoology, vol. 33, no. 2, pp. 103-106. http:// dx.doi.org/10.17582/pujz/2018.33.2.103.106.

SHAH, S.Z.H., AFZAL, M., KHAN, S.Y., HUSSAIN, S.M. and HABIB, R.Z., 2015 [viewed 14 December 2020]. Prospects of using citric acid as fish feed supplement. International Journal of Agriculture and Biology [online], vol. 17, pp. 1-8. Available from: http://www. fspublishers.org

SHAH, S.Z.H., FATIMA, M., AFZAL, M. and BILAL, M., 2021. Interactive effect of citric acid, phytase and chelated mineral on growth performance, nutrient digestibility and whole-body composition of Labeo rohita fingerlings. Aquaculture Research, vol. 52, no. 2, pp. 842-858. http://dx.doi.org/10.1111/are.14939.

SNEDECOR, G.W. and COCHRAN, W.G., 1991. Statistical methods. 8th ed. New York: Iowa State University Press, 503 p.

THIAM, S., FALL, J., LOUM, A., SAGNE, M. and DIOUF, M., 2015. Use of effective microorganisms (Em) in tilapia diets: effects of growth performance and carcass composition. International Journal of Current Microbiology and Applied Sciences, vol. 4, no. 11, pp. 536-549.

VON DANWITZ, A. and SCHULZ, C., 2020. Effects of dietary rapeseed glucosinolates, sinapic acid and phytic acid on feed intake, growth performance and fish health in turbot (Psetta maxima L.).Aquaculture, vol. 516, pp. 734624. http://dx.doi.org/10.1016/j. aquaculture.2019.734624.

YIGIT, N.O., BAHADIR KOCA, S., DIDINEN, B.I. and DILER, I., 2018. Effect of protease and phytase supplementation on growth performance and nutrient digestibility of rainbow trout (Oncorhynchus mykiss, Walbaum) fed soybean meal-based diets. Journal of Applied Animal Research, vol. 46, no. 1, pp. 29-32. http://dx.doi.org/10.1080/09712119.2016.1256292.

ZHU, Y., QIU, X., DING, Q., DUAN, M. and WANG, C., 2014. Combined effects of dietary phytase and organic acid on growth and phosphorus utilization of juvenile yellow catfish Pelteobagrus fulvidraco. Aquaculture, vol. 430, pp. 1-8. http://dx.doi. org/10.1016/j.aquaculture.2014.03.023. 